\title{
Evaluación de tres métodos para la inactivación de coliformes y Escherichia coli presentes en agua residual doméstica, empleada para riego
}

\author{
Naydú Rojas-Higuera ${ }^{1}$, Andrea Sánchez-Garibello ${ }^{1}$, Adriana Matiz-Villamil ${ }^{2}$, Juan Carlos Salcedo-Reyes ${ }^{3}$, \\ Ana Karina Carrascal-Camacho ${ }^{4}$, Aura M. Pedroza-Rodríguez ${ }^{1 *}$
}

${ }^{1}$ Laboratorio de Microbiología Ambiental y Suelos, ${ }^{2}$ Laboratorio de Biotecnología Aplicada, ${ }^{4}$ Laboratorio de Microbiología de Alimentos. Grupo de Biotecnología Ambiental e Industrial (GBAI). Departamento de Microbiología. ${ }^{3}$ Grupo de Películas Delgadas. Departamento de Física. Facultad de Ciencias. Pontificia Universidad Javeriana, Bogotá, D.C. Colombia.

*apedroza@yahoo.com

Recibido: 23-06-2010; Aceptado: 14-08-2010

\begin{abstract}
Resumen
Objetivo. Evaluar tres tratamientos (lagunaje, fotocatálisis con $\mathrm{TiO}_{2}$ y desinfección química) para la inactivación de coliformes totales y Escherichia coli presentes en agua residual doméstica empleada para riego agrícola. Materiales y métodos. El agua residual fue caracterizada por medio de análisis físicos, químicos y microbiológicos. Posteriormente fue sometida a un tratamiento de lagunaje facultativo(TLF), pos tratamiento fotocatalítico $\left(\mathrm{PTFTiO}_{2} / \mathrm{UV}\right.$ ) y pos tratamiento químico (PTQ NaClO). Valorando la capacidad desinfectante de cada uno de ellos para inactivar coliformes totales y E. coli. A continuación se procesaron tres nuevos lotes de agua residual y se utilizaron para realizar pruebas de riego a escala de laboratorio por 30 días, empleando como modelo plantas de Lactuca sativa variedad Batavia y evaluando la concentración inicial y final de los dos grupos. Resultados. El $\mathrm{PTFTiO}_{2} / \mathrm{UV}$ fue significativamente superior que TLFLF y el PTQ NaClO ( p<0,0001) obteniendo $100 \%$ de inactivación para coliformes y E. coli a los 30 minutos de irradiación a escala de reactor. Respecto a las pruebas de riego de L. sativa se demostró que al utilizar el agua tratada por $\mathrm{PTFTiO}_{2} / \mathrm{UV}$ no se presentó contaminación con $E$. coli y coliformes a los 30 días de proceso. Por el contrario en las plantas regadas con agua tratada por TLF y PTQ NaClO se observó un incremento en las dos poblaciones generando un problema de contaminación de las hortalizas al finalizar la prueba de laboratorio. Conclusión. La fotocatálisis heterogénea $\mathrm{TiO}_{2}$ fue un método eficaz para la reducción de coliformes y E.coli en aguas residuales domésticas.
\end{abstract}

Palabras clave: fotocatálisis heterogénea, calidad higiénica, Lactuca sativa

\begin{abstract}
Evaluation of three methods for the inactivation of coliforms and Escherichia coli present in domestic wastewaters used in irrigation. Objective. To evaluate three treatments (facultative stabilization ponds, heterogeneous photocatalysis with $\mathrm{TiO}_{2}$ and chemical disinfection with sodium hypochlorite) for the inactivation of coliforms and Escherichia coli present in domestic wastewaters used in agricultural irrigation. Materials and methods. Wastewater was characterized by physical, chemical and microbiological analyses and was then exposed to a facultative pond treatment (FPT), post-photocatalytic treatment $\left(\mathrm{PTFTiO}_{2} / \mathrm{UV}\right)$ and post-chemical treatment $(\mathrm{PTQNaClO})$ to assess the disinfecting capacity of each method in the inactivation of total coliforms and $E$. coli. Three new samples of wastewater were processed and used in irrigation tests on a laboratory-scale basis for 30 days, using Lactuca sativa cultivar. Batavia as a model plant and evaluating the initial and final concentrations of the two groups. Results. $\mathrm{PTFTiO} / \mathrm{UV}$ was significantly higher than $\mathrm{FPT}$ and $\mathrm{PTQNaClO}$ ( $<<0.0001$ ), obtaining $100 \%$ of inactivation of coliforms and E. coli after 30 minutes of irradiation at a reactor scale. Regarding the irrigation tests with $L$. sativa, we showed that using water treated by $\mathrm{PTFTiO}_{2} / \mathrm{UV}$ there is no contamination with $E$. coli and coliforms after 30 days. On the contrary, plants irrigated with water treated by FPT and PTQNaClO showed an increase in the two populations originating a
\end{abstract}


contamination problem in the vegetable by the end of the laboratory experiments. Conclusion. The heterogeneous photocatalysis with $\mathrm{TiO}_{2}$ was an effective method in the reduction of coliforms and $E$. coli present in domestic wastewater.

Key words: heterogeneous photocatalysis, hygienic quality, Lactuca sativa

\section{Resumo}

Avaliação de três métodos para a inativação de coliformes totais e Escherichia coli presentes em águas residuais domésticas utilizadas para a irrigação. Objetivo. Avaliar três tratamentos (lagoas, fotocatálise com $\mathrm{TiO}_{2}$ e desinfecção química) para inativação de coliformes totais e Escherichia coli presentes em águas residuais domésticas utilizadas na irrigação dos cultivos. Materiais e métodos. $\mathrm{O}$ efluente foi caracterizado por analises físicos, químicos e microbiológicos. Posteriormente foi submetido a tratamento de lagoas facultativas (TLF), pós-tratamento fotocatalítico $\left(\mathrm{PTF}_{\mathrm{TiO} / \mathrm{UV}}\right)$ e pós-tratamento químico $\left(\mathrm{PTQ}_{\mathrm{NaClO}}\right)$. Avaliando a capacidade desinfetante de cada um para inativar coliformes totais e E. coli. Posteriormente, foram processados três novos lotes de águas residuais para testar a irrigação em escala de laboratório por 30 dias, utilizando-se como modelo plantas de Lactuca sativa variedade Batavia e avaliando a concentração inicial e final dos dois grupos. Resultados. O PTFTiO / $/$ VV foi significativamente maior a TLFLF e PTQ NaClO (p<0,0001) apresentando $100 \%$ de inativação para coliformes e E. coli em 30 minutos de irradiação a escala do reator. Quanto aos testes de irrigação de $L$. sativa foi demonstrado que, ao utilizar a água tratada com $\mathrm{PTFTiO}_{2} / \mathrm{UV}$ não se apresentou contaminação com E. coli e coliformes a 30 dias do tratamento. Em contrapartida, em plantas irrigadas com água tratada por TLFe PTQ NaClO observou-se um aumento nas duas populações gerando um problema de contaminação de hortaliças no final do teste de laboratório. Conclusão. A fotocatálise heterogênea TiO ${ }_{2}$ foi um método eficaz para reduzirem coliformes e $E$. coli em águas residuais domésticas.

Palavras-chave: foto-catálise heterogênea, qualidade higiênica, Lactuca sativa

\section{Introducción}

En los últimos 10 años, los agricultores han incorporado en sus prácticas agrícolas el uso de aguas recicladas, debido a que los recursos hídricos naturales paulatinamente se están reduciendo (1), este tipo de agua constituye un riesgo al traer microorganismos patógenos que pueden llegar a los cultivos (2). Parte de esta agua proviene de lagunas de oxidación, donde se han realizado tratamientos primarios para reducir la carga orgánica (3). La sabana de Bogotá, es una de las fuentes de hortalizas más grandes de país, es frecuente el uso de estas aguas de riego recicladas. Sin embargo, a pesar de tener un tratamiento biológico previo, no se garantiza la calidad microbiológica debido a la carga biológica tan elevada que ingresa a las lagunas de oxidación de modo que, si bien se logra reducir la concentración de bacterias, no es apta para riego. Tradicionalmente se utiliza la desinfección con cloro para eliminar microorganismos en aguas residuales y potables; por su bajo costo, facilidad de adquisición y efectividad comprobada. No obstante, este método químico tiene desventajas como su inactivación en presencia de materia orgánica, generación de olores, posible formación de intermediarios tóxicos como cloraminas y trihalometanos (4). Por estas razones recientemente, se han desarrollado otras alternativas de inactivación como los procesos de oxidación avanzada (POAs), dentro de los que se destaca la fotocatálisis heterogénea con $\mathrm{TiO}_{2}$. El efecto desinfectante o esterilizador de un semiconductor (catalizador) se basa en la generación de pares electrón-hueco y su posterior recombinación en procesos de transferencia de carga interfacial (oxidativos o reductivos), en presencia de radiación electromagnética con energía mayor que la brecha de energía prohibida del semiconductor $\left(\mathrm{E}_{\mathrm{g}} \sim 3.4 \mathrm{eV}\right)$. Se ejerce, de esta manera, un efecto inactivador sobre la población microbiana expuesta mediante la oxidación del agua adsorbida en la superficie del $\mathrm{TiO}_{2}$ para formar especies fuertemente oxidantes, como los radicales hidroxilo $(* \mathrm{OH})$, que son considerados los principales responsables del daño celular en los microorganismos $(5,6)$.

El objetivo de este trabajo fue evaluar la efectividad de tres tratamientos: Biológico (lagunaje facultativo), físico $\left(\mathrm{TiO}_{2} / \mathrm{UV}\right)$ y químico $(\mathrm{NaClO})$ como alternativas para la reducción o eliminación de coliformes totales y Escherichia coli presentes en agua residual doméstica empleada para riego de Lactuca sativa variedad Batavia.

\section{Materiales y métodos}

\section{Muestreo de agua residual}

El agua residual doméstica se obtuvo de una planta de tratamiento ubicada a $35 \mathrm{Km}$ de Bogotá; región que cuenta con una temperatura promedio de $13{ }^{\circ} \mathrm{C}$, una precipitación anual de $890 \mathrm{~mm}$ y se encuentra a una altura que oscila entre los $2600 \mathrm{~m}$ sobre el nivel del mar (7).

Respecto a la caracterización inicial se realizaron determinaciones para demanda biológica de oxígeno al quinto día $\left(\mathrm{DBO}_{5}\right)$, demanda química de oxígeno (DQO), (Método 5210B) (8), sólidos suspendidos totales (SST) 
(Método 2540 D), (7) sólidos disueltos totales (SDT) (Método 2540 C) (8), sólidos sedimentables (SS) y sólidos totales (ST), unidades de color (UC), materia orgánica (MO), fósforo total $\left(\mathrm{F}_{\mathrm{T}}\right)$ (Método 4500-P B y E), (8) y nitrógeno total $\left(\mathrm{N}_{\mathrm{T}}\right),(8)$. Adicionalmente, se realizó recuento de coliformes totales y Escherichia coli en agar Chromocult (Método 9223), (8).

\section{Tratamiento de lagunaje facultativo (TLF)}

El tratamiento de lagunaje facultativc o TLF consistió en una planta de tratamiento de aguas residuales (PTAR) la cual estaba compuesta por un pre tratamiento (rejillas de cribado), tratamiento primario (desarenadores y trampa de grasa) y lagunas biológicas (aeróbica, facultativa primaria y facultativa secundaria). Con un tiempo de retención de 24 horas. Para evaluar la eficiencia del TLF se realizaron muestreos iniciales y finales en los puntos en los que ingresa y sale el agua de la PTAR, realizando análisis físicos, químicos y microbiológicos.

\section{Pos tratamiento fotocatalítico con $\mathrm{TiO}_{2}$ (PTFTiO $/$ /UV)}

\section{Elaboración de películas de dióxido de titanio}

Se utilizaron láminas de vidrio de ( $20 \mathrm{~mm}$ x $26 \mathrm{~mm})$ como sustrato, se lavaron y desengrasaron con agua-etanolacetona-agua (9). La superficie del $\mathrm{SiO}_{2}$ se modificó con $\mathrm{H}_{2} \mathrm{O}_{2} / \mathrm{H}_{2} \mathrm{SO}_{4} 3: 1$ por 20 minutos (9). Las películas se crecieron por inmersión simple en solución de $\mathrm{TiO}_{2}$ USP al $1 \%(\mathrm{~m} / \mathrm{v})$ y $500 \mu \mathrm{l} \mathrm{de} \mathrm{HNO}_{3}(\mathrm{pH} 1,3)$. Se sedimentaron por 8 horas a $50^{\circ} \mathrm{C}$ y se calcinaron a $450^{\circ} \mathrm{C}$ por 1 hora (2). La superficie de las películas se observó por microscopía electrónica de barrido y se realizó difracción de rayos X (9). Adicionalmente, para valorar la adhesión del $\mathrm{TiO}_{2}$ al sustrato se le realizó una prueba de desprendimiento que consistió en sonicar la películas crecidas a 50 y $450{ }^{\circ} \mathrm{C}$ por 15 minutos; se dejaron secar a $20{ }^{\circ} \mathrm{C}$ y nuevamente se determinó el peso de $\mathrm{TiO}_{2}$ descontando el peso de la lámina de vidrio (9).

El sistema fotocatalítico o pos tratamiento $\left(\mathrm{PTFTiO}_{2} / \mathrm{UV}\right)$ estaba compuesto por tres reactores cilíndricos de cuarzo con un volumen total de $200 \mathrm{ml}$, los cuales fueron operados al $60 \%$ de su capacidad. Cada uno de ellos estaba colocado sobre un agitador horizontal a $100 \mathrm{rpm}$, contenía 4 películas de dióxido de titanio ( $249 \mu \mathrm{g} \mathrm{TiO} / \mathrm{mg}$ de sustrato), sensor de temperatura y puertos de muestreo para retirar el agua tratada. Externamente se instalaron 2 lámparas de $15 \mathrm{~W}$. A cada rector se le adicionaron $120 \mathrm{ml}$ del agua residual pre tratada por el TLF con poblaciones iniciales de coliformes totales y E. coli de $4 \mathrm{U} \log$ y $3 \mathrm{U} \log$. Después de un periodo de estabilización por 30 minutos en oscuridad se inició la irradiación durante 30 minutos; al finalizar el proceso se cuantificaron las poblaciones de E. coli y coliformes totales.

\section{Pos tratamiento químico con cloración (PTQNaClO)}

El sistema químico consistió en tres reactores de vidrio de $1000 \mathrm{ml}$ que contenían $548,13 \mathrm{ml}$ del agua residual pre tratada con el TLF y $1,87 \mathrm{ml}$ de hipoclorito de sodio (200 ppm). La solución desinfectante se dejó reaccionar con el agua por 8 horas a temperatura de $20^{\circ} \mathrm{C}$ y se realizaron recuentos de coliformes y $E$. coli.

Para determinar diferencias significativas entre tratamientos se realizó una comparación de medias por medio de una prueba de Turkey con el programa estadístico SAS 6.0 para Windows.

\section{Evaluación de la calidad microbiológica del agua residual tratada empleando como modelo de riego a Latuca sativa var Batavia a escala de laboratorio.}

Se realizaron tres nuevos montajes para cada uno de los tratamientos (TLF, $\mathrm{PTFTiO}_{2} / \mathrm{UV}$ y $\mathrm{PTQNaClO}$ ) los cuales fueron muestreados en los tiempos de retención hidráulica correspondientes a cada tratamiento. El experimento se realizó usando 80 plantas de Lactuca sativa var. Batavia con cuatro semanas de desarrollo y 5 hojas verdaderas. Se realizó un análisis microbiológico inicial sembrando en agar Chromocult para determinar la presencia de coliformes totales y $E$. coli en la zona aérea, radicular y en el suelo. Posteriormente, las plantas se sembraron en $30 \mathrm{~g}$ de suelo abonado estéril; se adicionaron diariamente $4 \mathrm{ml}$ de agua desinfectada por cada tratamiento y como control se utilizó agua destilada esterilizada a $121^{\circ} \mathrm{C}, 151 \mathrm{~atm}$., por 15 minutos. A los 30 días, se recolectaron hojas, raíces y suelo para realizar los mismos recuentos que al inicio del experimento. Se realizó una comparación de medias entre tratamientos tomando como variable dependiente las unidades formadoras de colonia expresadas como logaritmo en base 10 (U log) y se utilizó el programa estadístico SAS 9.0.

\section{Resultados}

\section{Tratamiento de lagunaje facultativo (TLF)}

El TLf removió eficientemente algunos de los parámetros físico-químicos como sólidos sedimentables SS (100\%), 
demanda bioquímica de oxígeno $\mathrm{DBO}_{5}(>30 \%$ de remoción en carga para residuales domésticas generados por usuarios existentes) y demanda química de oxígeno DQO (>30\% de remoción en carga). Cumpliendo así con los límites básicos de vertimiento a un cuerpo de agua para residuales domésticos según el artículo 72 del decreto 1594/ 1984 del Ministerio de Salud. No obstante, algunas de las determinaciones realizadas en este estudio no están contempladas en el decreto y se observó que los porcentajes de remoción son bajos, generando un incremento en la concentración de nutrientes responsables de los problemas de eutroficación, aspecto coloreado y presencia de coliformes totales y E. coli. Es el caso de la $\mathrm{MO}(48 \%), \mathrm{F}_{\mathrm{T}}$ $(25 \%), \mathrm{N}_{\mathrm{T}}(0 \%)$, SST (32\%), UC (6\%) y poblaciones de coliformes totales y E. coli de 4 y $3 \mathrm{U} \log$. De acuerdo con los resultados obtenidos el TLF que asoció sistema primario y biológico, no fue suficiente para eliminar los microorganismos responsables de la contaminación de aguas para uso agrícola y para ciertos parámetros los valores de vertimiento fueron elevados (Tabla 1).

\section{Pos tratamiento fotocatalítico con $\mathrm{TiO}_{2}$ (PTFTiO,/UV)}

\section{Elaboración de las películas y caracterización}

Con relación a las características superficiales de las películas de $\mathrm{TiO}_{2}$ se observó en las fotografías de microscopía electrónica de barrido que presentan una superficie rugosa, irregular, porosa y no tienen un tamaño de grano homogéneo. El cual varió entre 7,5 y 12,5 $\mu \mathrm{m} \pm$ 1,87. Sin embargo, al calcinarlas por 1 hora a $450{ }^{\circ} \mathrm{C}$ se mejoró sustancialmente la homogeneidad, adhesión al sustrato y se presentó menor desprendimiento del óxido semiconductor. En las películas secadas a $50^{\circ} \mathrm{C}$ la cantidad de $\mathrm{TiO}_{2}$ depositado fue de $94 \mu \mathrm{g} / \mathrm{mg}$ y al finalizar la prueba de sonicación la cantidad de $\mathrm{TiO}_{2}$ residual fue de 4,5 $\mu \mathrm{g}$ / $\mathrm{mg}$. Por el contrario en las películas calcinadas la concentración inicial del $\mathrm{TiO}_{2}$ fue de $30,6 \mu \mathrm{g} / \mathrm{mg}$ y al finalizar la sonicación el valor fue de $30,5 \mu \mathrm{g} / \mathrm{mg}$ lo que equivale a un porcentaje de desprendimiento del $0,32 \%$ valor más bajo que el obtenido a $50{ }^{\circ} \mathrm{C}$ que fue del $95 \%$ (Figura 1).

La figura 2 presenta el patrón de difracción de rayos $\mathrm{X}$ para una película calcinada a $450^{\circ} \mathrm{C}$, en la cual se observan los picos $27,4^{\circ}$ y $36,08^{\circ}$ que corresponder a los planos (101), (110) para rutilo. Para anatasa se presentaron los picos $25,28^{\circ}, 37,80^{\circ}$ equivalentes a los planos (101) y (004).

\section{Pruebas de inactivación fotocatalítica a escala de laboratorio}

Al evaluar el potencial desinfectante de la fotocatálisis heterogénea con $\mathrm{TiO}_{2}\left(\mathrm{PTFTiO}_{2} / \mathrm{UV}\right)$ como pos tratamiento al TLF se observó que a los 30 minutos de irradiación los porcentajes de inactivación para E. coli y coliformes totales fueron del $100 \%$ con un $\mathrm{pH}$ final de 8,2 . Partiendo de unas concentraciones iniciales de 3 y 4 unidades logarítmicas $\left(\mathrm{U} \log _{10}\right)$ para cada población (Figura 3).

Tabla 1. Caracterización física, química y microbiológica del agua residual entrada y salida de PTAR.

\begin{tabular}{|c|c|c|c|c|}
\hline Parámetro & $\begin{array}{l}\text { Entrada } \\
\text { PTAR }\end{array}$ & $\begin{array}{l}\text { Salida } \\
\text { PTAR }\end{array}$ & $\begin{array}{c}\text { Remoción } \\
\%\end{array}$ & $\begin{array}{c}\text { Decreto 1594/84 } \\
\text { Límite de vertimientos }\end{array}$ \\
\hline Materia orgánica mg/L & 221 & 114 & 48 & ND \\
\hline Fósforo Total mg/L P & 4,08 & 3,06 & 25 & ND \\
\hline Nitrógeno Total mg/L N & 30 & 43 & 0 & ND \\
\hline SST mg/L & 162 & 111 & 32 & 100 \\
\hline $\mathrm{SS} \mathrm{Cm} / \mathrm{L} / \mathrm{h}$ & 1,2 & 0 & 100 & $2 \mathrm{ml} / \mathrm{L}$ \\
\hline $\mathrm{DBO}_{5} \mathrm{mg} / \mathrm{LO}_{2}$ & 263 & 162 & 38 & 60 \\
\hline $\mathrm{DQO} \mathrm{mg} / \mathrm{L} \mathrm{O}_{2}$ & 589 & 304 & 48 & 120 \\
\hline Unidades de color & 283,7 & 267,8 & 6 & 50 \\
\hline $\mathrm{pH}$ & 7,6 & 7,4 & & $5-9,0$ \\
\hline E. coli $\mathrm{UFC} / \mathrm{mL}$ & $6 \times 10^{3}$ & $1 \times 10^{3}$ & 83 & $\longrightarrow$ \\
\hline Coliformes UFC/mL & $1 \times 10^{5}$ & $1 \times 10^{4}$ & 90 & $5000 \mathrm{NMP} / 100 \mathrm{~mL}$ \\
\hline
\end{tabular}




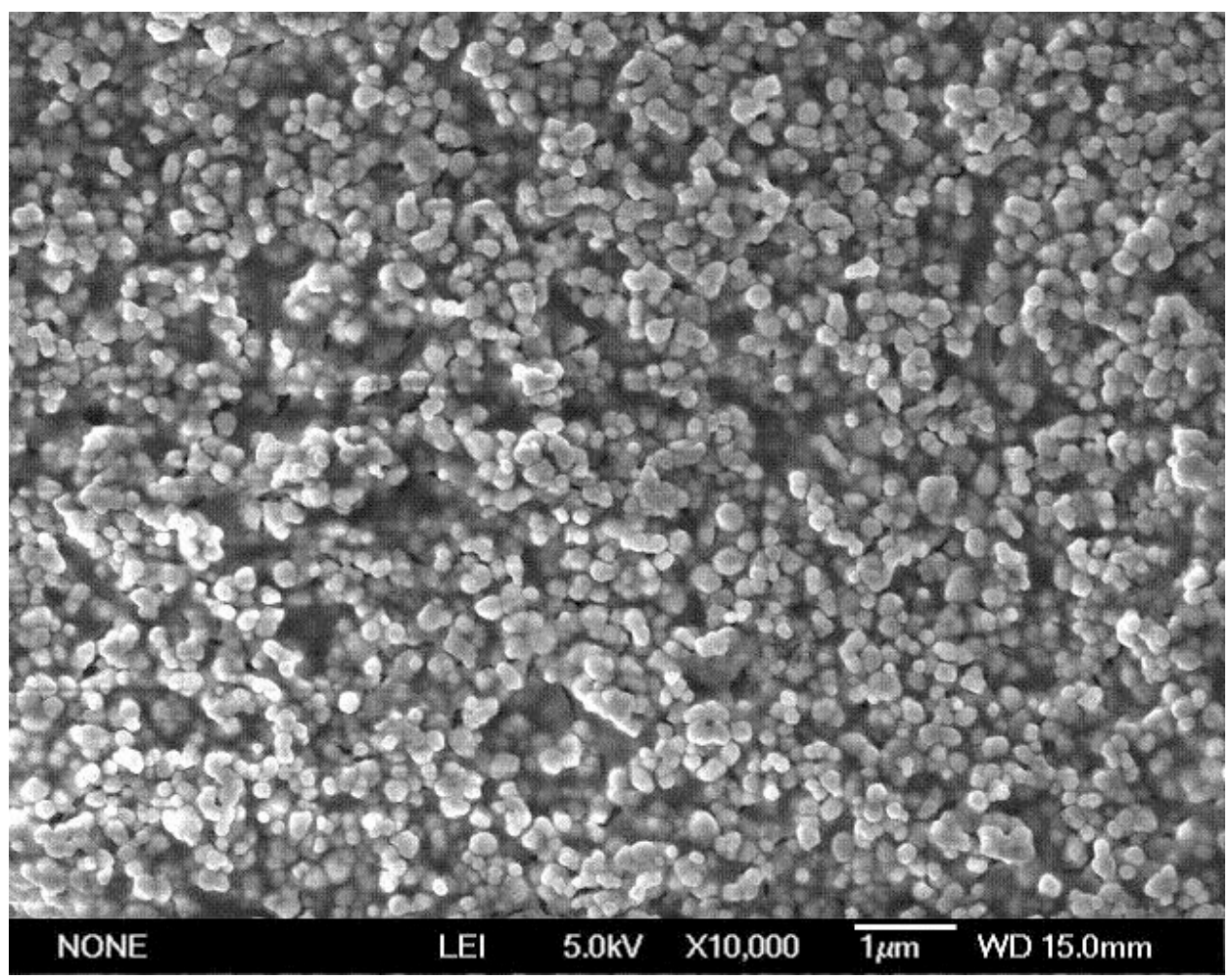

A

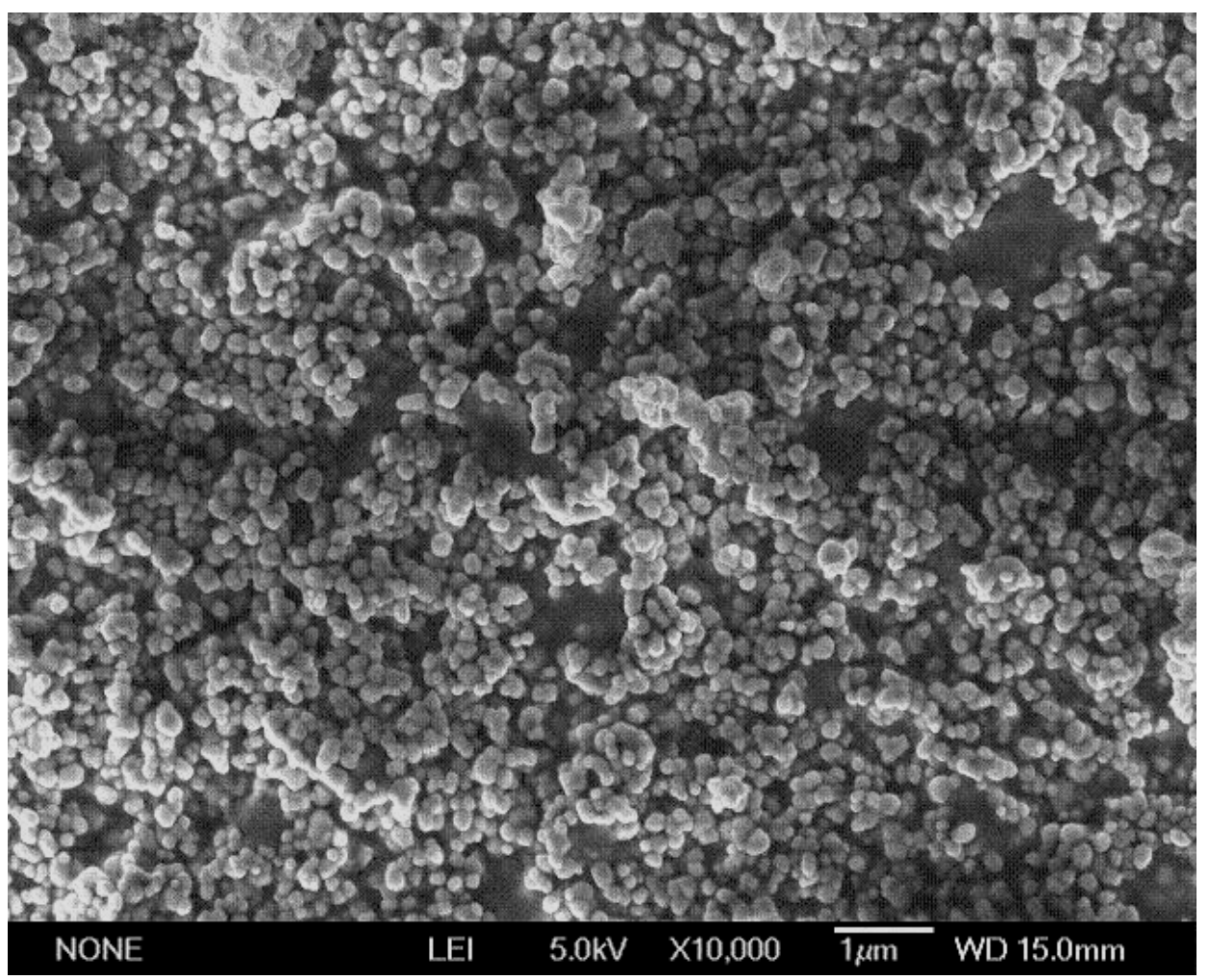

B

Figura 1. Microscopía electrónica de barrido para películas de $\mathrm{TiO}_{2}$ crecidas por sedimentación simple. (a) calcinación a $450{ }^{\circ} \mathrm{C}$. (b) secado a $50^{\circ} \mathrm{C}$ 


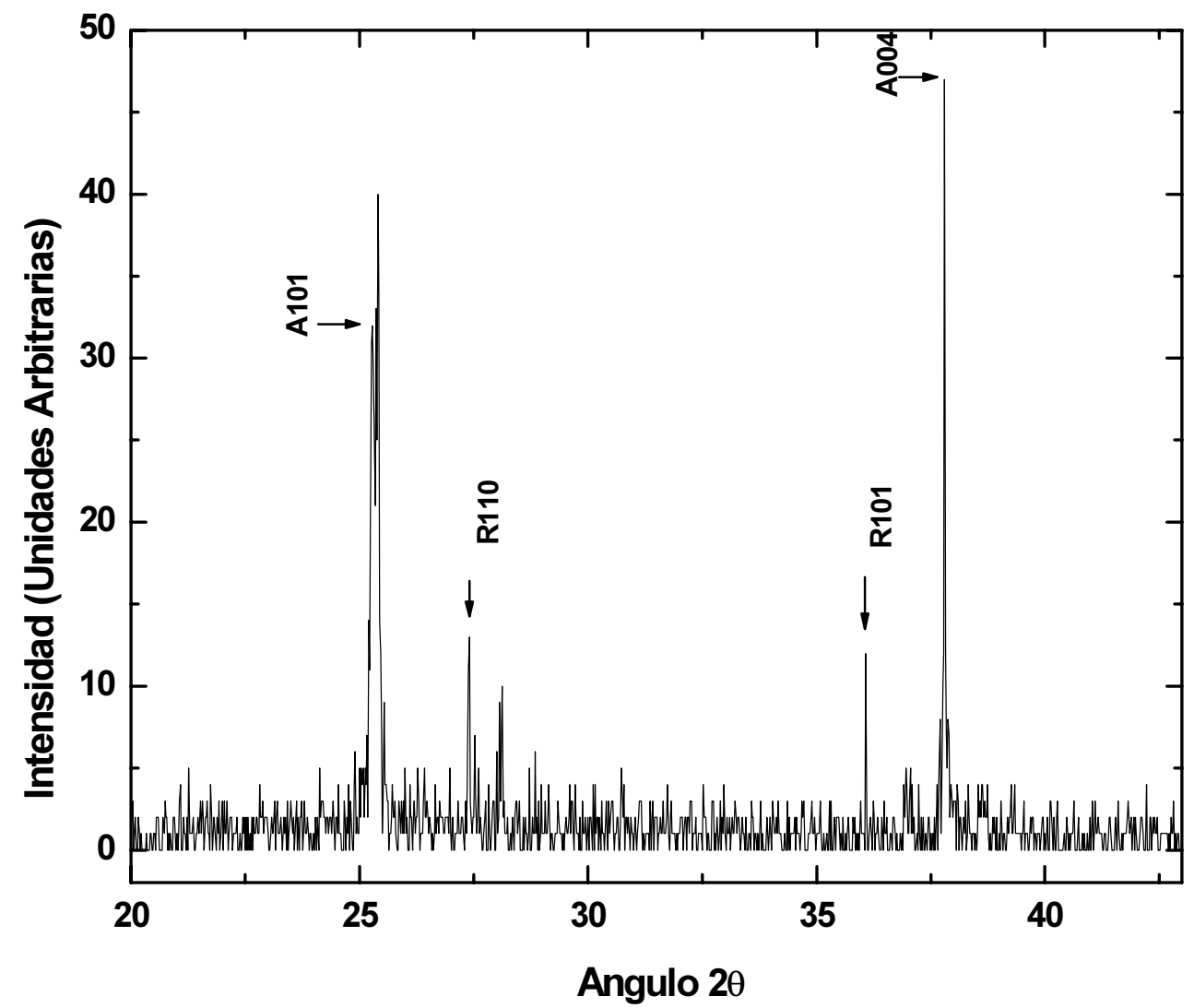

Figura 2. Patrón de difracción de la película de $\mathrm{TiO}_{2}$. (A) anatasa, (R) rutilo.

\section{Pos tratamiento químico por cloración (PTQNaClO)}

El sistema de desinfección química a las 8 horas de tratamiento redujo en $0,17 \mathrm{U} \log _{10}$ los coliformes totales y en $0,69 \mathrm{U} \log _{10}$ a $E$. coli $\mathrm{pH} 8,0$. Valores que no superan a los obtenidos con $\mathrm{PTFTiO}_{2} / \mathrm{UV}$ ya que con este sistema se presentó una efectividad del $100 \% 16$ veces más rápido que por cloración (Figura 3).

\section{Evaluación de la calidad microbiológica del agua residual tratada empleando como modelo de riego a Latuca sativa var Batavia a escala de laboratorio}

Los recuentos finales de los tres nuevos lotes para coliformes totales fueron de 4,4 $\mathrm{U} \log _{10}$ en el TLF, 3,1 U $\log _{10}$ para PTQ $\mathrm{NaClO}$, y $0 \mathrm{U} \log _{10}$ para PTQ, en el caso de E. coli fueron de $2,8 \mathrm{U} \log _{10}, 2,2 \mathrm{U} \log _{10}$ y $0 \mathrm{U} \log _{10}$ para cada uno de los tratamientos analizados.
En las plantas de L. sativa var Batavia inicialmente se encontró una concentración de coliformes totales de 4,1, 5,18 y $6 \mathrm{U} \log _{10}$ en la zona aérea, en el suelo y en la zona radicular respectivamente. E. coli no se detectó en el material vegetal.

Al finalizar los 30 días de riego con agua residual tratada por los tres sistemas, se presentaron diferencias altamente significativas entre tratamientos $(\mathrm{p}<0,0001)$ demostrando que el pos tratamiento fotocatalítico $\left(\mathrm{PTFTiO}_{2} / \mathrm{UV}\right)$ fue superior que el TLF y el PTQNaClO en cuanto a la inactivación de $E$. coli debido a que no se recuperó el microorganismo en ninguna de las muestras analizadas (Figura 4). En el PTQNaClO, se encontró una concentración de 0,33 en zona aérea, 0,27 en zona radicular y $0,40 \mathrm{U} \log _{10}$ en suelo para E. coli. Finalmente con el TLF se recuperaron $1,1,0,63$ y $1,4 \mathrm{U} \log _{10}$ en las mismas zonas de muestreo. En el control con agua destilada no se recuperaron colonias de E. coli en ninguna de las muestras analizadas. 


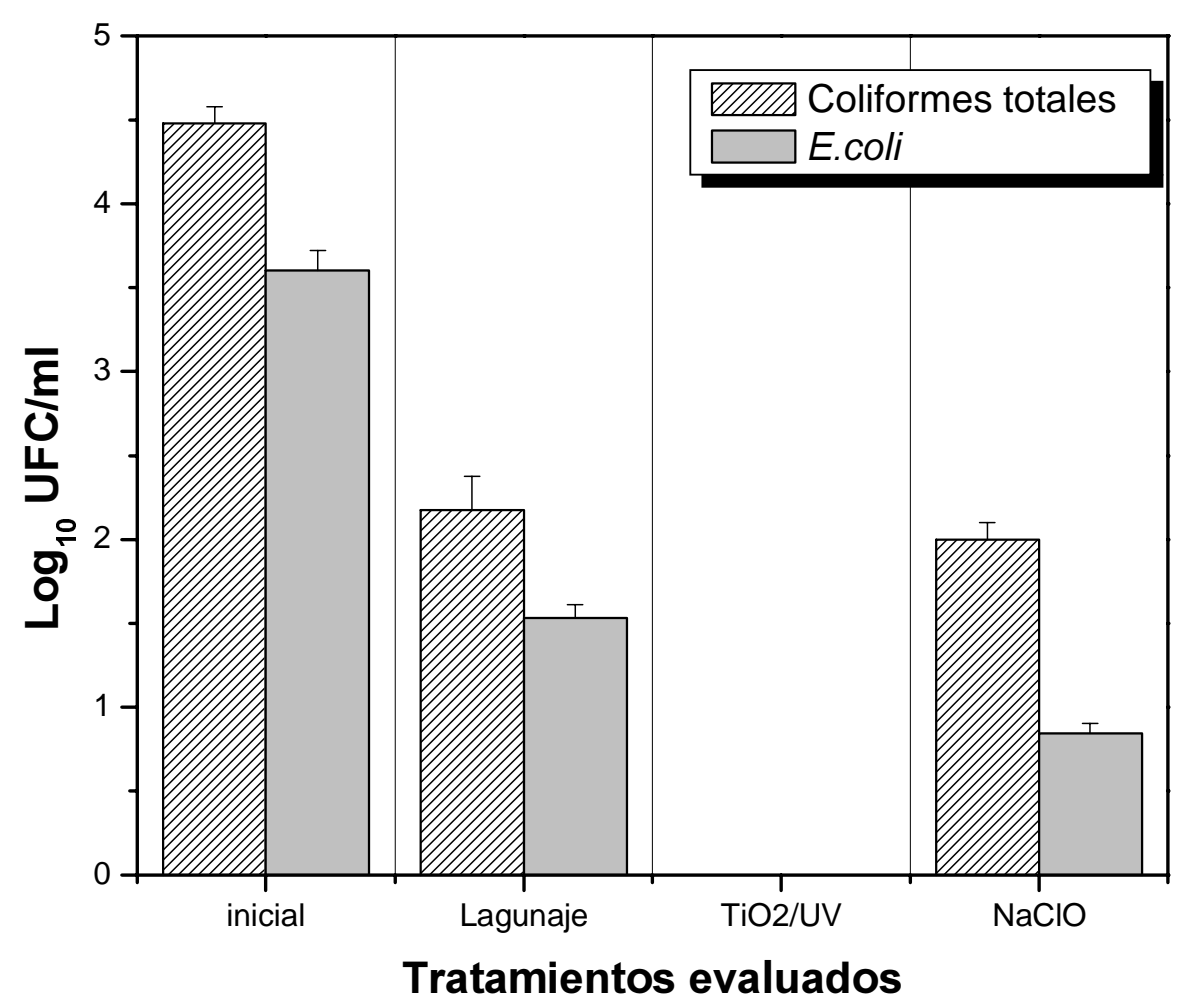

Figura 3. Reducción de coliformes y E. coli en los tres tratamientos en estudio

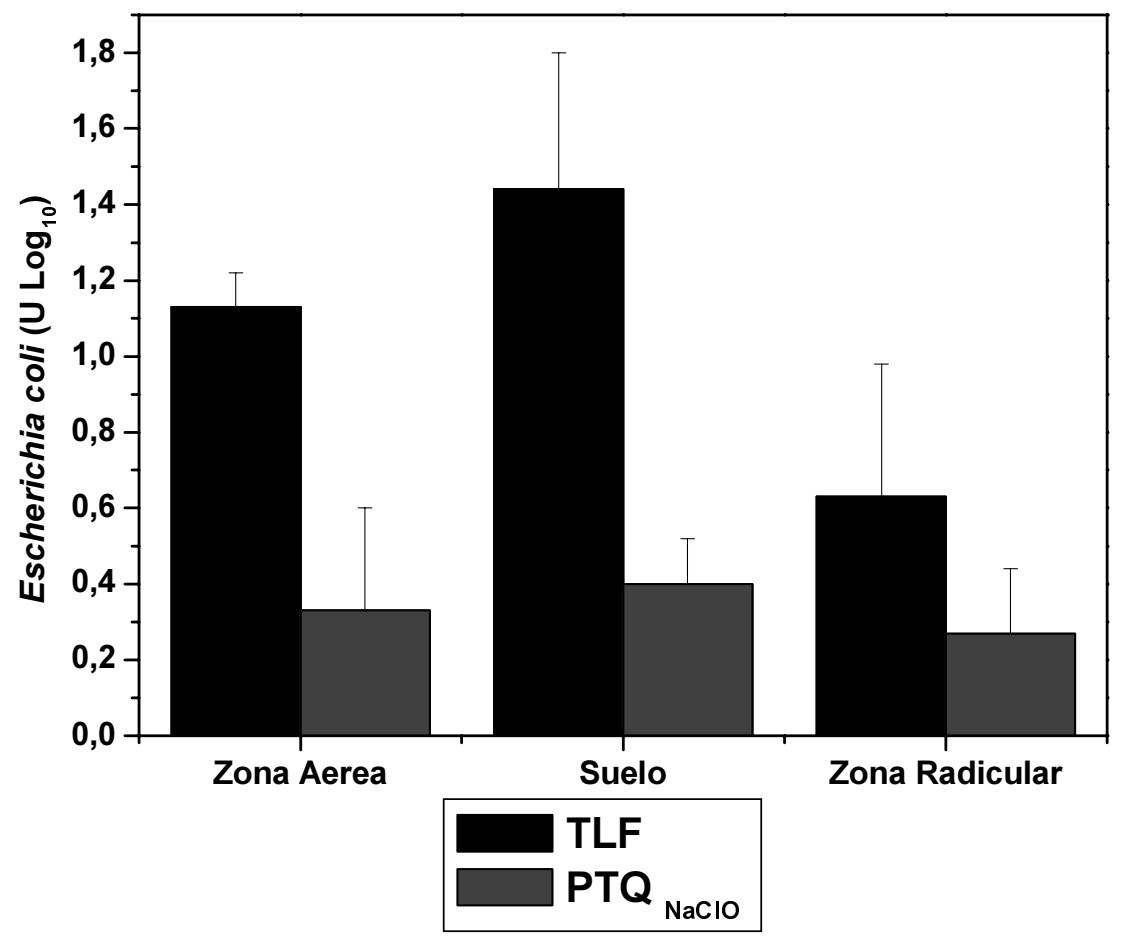

Figura 4. Reducción E. coli de acuerdo con la distribución espacial en las plantas de Lactuca sativa a los 30 días de riego con agua tratada por los tres sistemas (TLF, PTQ $\mathrm{NaClO}$ ). En el PTF $\mathrm{TiO}_{2} \mathrm{UV}$ y el control con agua destilada no se recuperó $E$. coli. 
En cuanto al comportamiento de los coliformes totales se observó que la fotocatálisis heterogénea fue significativamente mejor que los demás métodos $(\mathrm{p}<0,0001)$ ya que al tratar el agua con este sistema no se recuperó ningún coliforme y a los 30 días de regadío los microorganismos que se aislaron $(0,21,1,1$ y 0,32 en zona aérea, radicular y suelo) correspondían posiblemente a las poblaciones iniciales de la matriz suelo, hoja y raíz. Estos datos se confirmaron al analizar los resultados del control con agua destilada en los cuales también se obtuvieron recuentos de 1,7, 4 y 2,1 U $\log _{10}$ en hojas, raíz y suelo. El TLF fue ineficiente para remoción de este grupo indicador ya que presentaron recuentos de 5,8 $\mathrm{U} \log _{10}$ en suelo, 5,5 $\mathrm{U} \log _{10}$ en la zona radicular y $4,3 \mathrm{U} \log _{10}$ en la zona aérea. Con el
PTQNaClO los recuentos finales fueron de 1,63, 1,8 y 1,8 en hoja, raíz y suelo (Figura 5).

\section{Discusión}

El uso de aguas recicladas para la agricultura ha ido en aumento en los últimos años, especialmente en países donde este recurso hídrico escasea (1, 3, 10). La calidad microbiológica de este tipo de agua está relacionada fundamentalmente con la calidad del tratamiento, el tiempo de retención hidráulica, la concentración de materia orgánica entre otros (11). Se ha señalado que aguas de

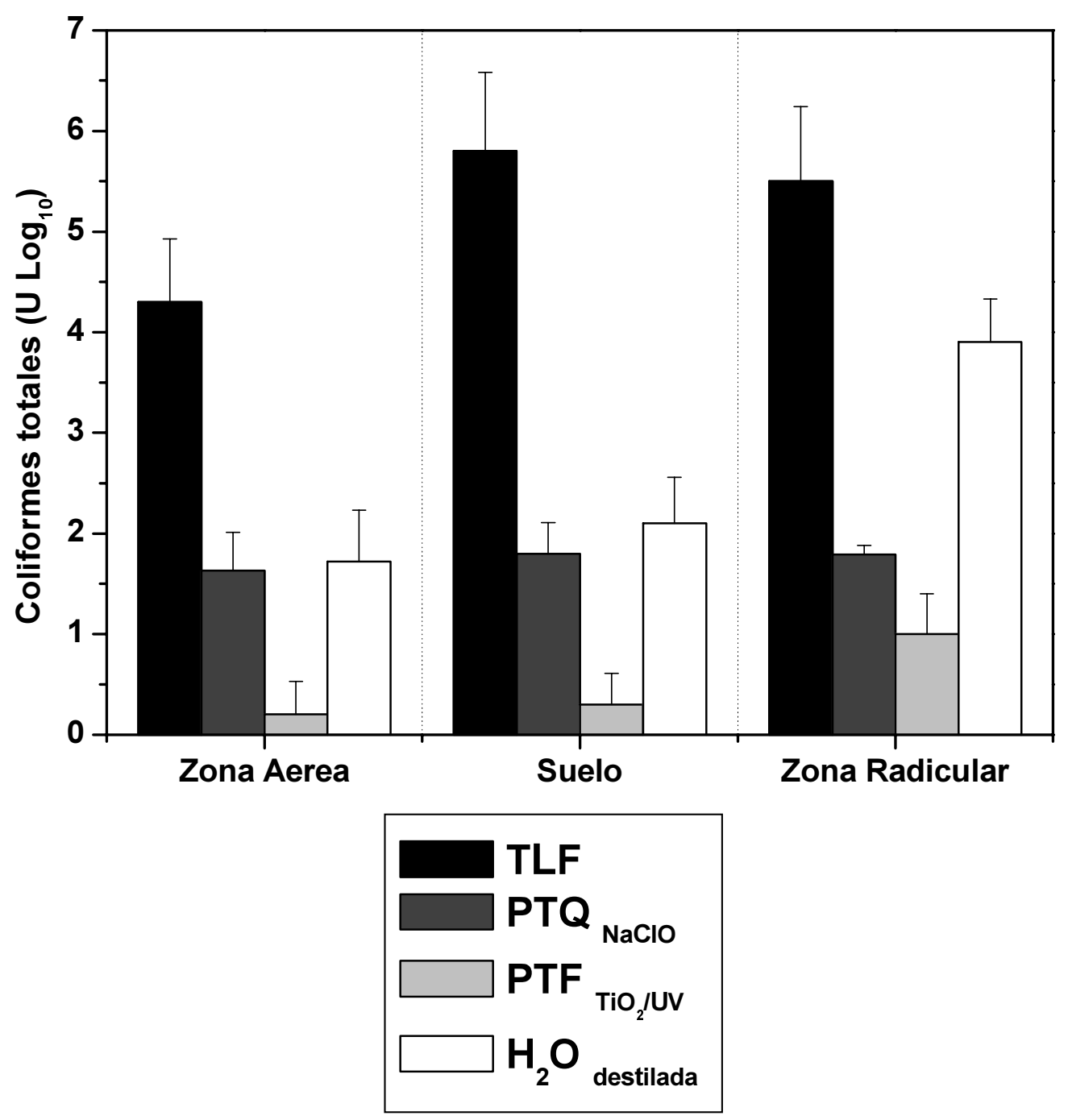

Figura 5. Reducción coliformes totales de acuerdo con la distribución espacial en las plantas de Lactuca sativa a los 30 días de riego con agua tratada por los tres sistemas (TLF, PTFTiO UV, PTQ NaClO y el control con agua destilada). 
baja calidad pueden ser vehículos de transmisión de microorganismos como Shigella, virus de la hepatitis A, Entamoeba histolytica entre otros (12). Generalmente las aguas recicladas utilizadas frecuentemente se someten a un solo tratamiento, donde no se incluye un paso específico de desinfección, en el caso de los coliformes donde inicialmente pueden existir poblaciones de $10^{8} \mathrm{ufc} / \mathrm{ml}$, los tratamientos primarios logran reducir 1-3 orden de magnitud (13), de tal manera que la presencia de coliformes suele ser superior a los estándares internacionales y nacionales resultados que se confirmaron en este estudio, además pueden encontrarse patógenos como Salmonella $(11,14)$. Koivunen (15) señala que la contaminación microbiana que reciben las aguas naturales está relacionada con la eficacia del tratamiento previo para remover materia orgánica, sólidos suspendidos y fósforo. El papel de los sólidos suspendidos se relaciona con la concentración de microorganismos entéricos, de ahí la importancia de realizar procesos de floculación. Los procesos de lagunaje son particularmente eficientes para eliminar coliformes fecales comparados con otros tratamientos, siempre que se presenten tiempos de retención largos (60 días), $\mathrm{pH}$ altos resultado de la fotosíntesis, inactivación por luz solar y predación por protozooarios; en este caso el tiempo de retención fue de 24 horas lo que impide su efecto microbicida (1). Por otro lado se sabe que los procesos de lagunaje no son eficientes en la remoción de la $\mathrm{DBO}_{5}$ (3), lo cual incide directamente en la presencia de microorganismos (15).

Esto indica la importancia de realizar un tratamiento complementario al sistema de lagunaje para mejorar la calidad bacteriológica, física y química del efluente (13). El proceso químico más utilizado es la aplicación de cloro por su bajo costo. Sin embargo, este puede verse afectado por factores como: Concentración de materia orgánica, concentración de cloro y tipo de microorganismos presentes; reduciendo su efectividad (4). En esta investigación se pudo establecer el impacto que tiene la presencia de carga orgánica en el agua ya que fueron necesarias 8 horas de contacto para poder reducir por encima del $90 \%$ la población de coliformes y E. coli. De otro lado su principal problema radica en la posible formación de compuestos como trihalometanos (compuesto cancerígenos), resultantes de la reacción del cloro con materia orgánica (16), constituyéndose en un riesgo químico. Esto ha generado que nuevas tecnologías como la fotocatálisis heterogénea con $\mathrm{TiO}_{2}$, surjan como alternativa para reducir los niveles de microorganismos patógenos e indicadores en aguas de uso agrícola $(2,5)$.

La efectividad lograda durante $\mathrm{PTFTiO}_{2} / \mathrm{UV}$ se debió principalmente a la conformación estructural del $\mathrm{TiO}_{2}$, debido a la presencia de la fase anatasa como se invidencia en la figura 2, siendo esta más fotoactiva que la fase rutilo por su alta cristalinidad lo cual le confiere pocos defectos en cuanto a la recombinación de electrones y huecos fotogenerados (17) los cuales producen especies reactivas del oxígeno (ROS) en contacto con el agua y aire del medio causando así un efecto de inactivación sobre $E$. coli donde los mecanismos que explican esta inactivación incluyen: Un daño parcial sobre la permeabilidad de la membrana externa, seguido por un daño a nivel de pared y en membrana citoplasmática causando la lísis celular (12). También se forman iones superóxidos $\left(\mathrm{O}_{2}\right)$ ó $\left(\mathrm{HO}_{2} \mathrm{O}\right)$ los cuales son menos efectivos contra las bacterias, debido a que las cargas negativas previenen que penetren las membranas celulares de las bacterias, no obstante causan un deterioro complementario en la célula (18). Estudios realizados por Nadtochenko, 2005, (19) permiten señalar que durante el proceso de fotocatálisis se dan los siguientes pasos: 1 . Cambios en la peroxidación del lipopolisacárido de la pared durante el inicio. 2. Formación de $\alpha$ y $\beta$ aldehídos insaturados durante el rompimiento de hidroperóxidos o endoperóxidos y la aparición de puentes de C-O durante la formación de grupos carboxi 3. También se presentan cambios importantes en el perfil de las bandas $\mathrm{PO}_{2}$ y 4 . Formación de bandas de amida que varían en función de la radiación, todos estos cambios generan la destrucción de E. coli (19).

Muchas variables afectan la fotocatálisis con $\mathrm{TiO}_{2}$, por ejemplo parámetros intrínsecos como el tamaño de partícula, el area superficial del semiconductor, método de preparación y estructura cristalina. asi como parámetros extrínsecos como la temperatura de reacción, la intensidad de la luz y el pH de la solución (20). Un aspecto importante a señalar es que la fotocatálisis puede ser eficaz contra la inactivación de bacterias aún en presencia de materia orgánica, caso que se dió en esta investigación donde la $\mathrm{DBO}_{5}$ evidenció la presencia de materia orgánica, concordando con los resultados de Rizzo, 2009 (10).

Es muy importante señalar que la fotocatálisis fue efectiva al destruir toda la población de E. coli en 30 minutos, concordando con los datos de Caballero, 2009 (21), evitando de esta manera la posible sobrevivencia de subpoblaciones resistentes, fenómeno que se pudo presentar en el tratamiento con cloro, donde después de un tratamiento con 200 ppm durante 8 horas quedó un porcentaje de sobrevivientes que dentro de un ecosistema como el agua puede ser un problema a largo plazo.

Con relación a los datos obtenidos en el cultivo de $L$. sativa, para el agua pos tratada por fotocatalisis no se presentó contaminación con E. coli, al finalizar el periodo de riego de las plantas, señalando la importancia de este tratamien- 
to en la inactivación de esta bacteria. Caso contrario sucedió en el tratamiento con hipoclorito de sodio en el cual las plantas resultaron contaminadas al entrar en contacto con el agua tratada ya que esta contenía $E$. coli. Para el tratamiento convencional la concentración de $E$. coli fue superior a los otros dos tratamientos indicando que el sistema lagunaje es una fuente directa de contaminación en los cultivos.

Es importante señalar que inicialmente la zona radicular, aérea y el suelo presentaron coliformes. Que a pesar de realizar el proceso de desinfección del material vegetal y la esterilización del suelo; siguieron presentes e incrementaron en algunos tratamientos como el control con agua destilada y fotocatálisis. Para los primeros el incremento se relacionó con el crecimiento normal de la población y con un fenómeno de percolación o escorrentía del agua a través del material vegetal hasta llegar al suelo que determinó la obtención de los recuentos más altos en el suelo. Un mecanismo similar se pudo presentar en el tratamiento fotocatalítico en el cual se recuperaron coliformes a los 30 dias del tratamiento. Estos microorganismos nuevamente provenían del material vegetal y no fueron aportados por el agua pos tratada. Finalmente, el uso de fotocatálisis heterogénea con $\mathrm{TiO}_{2}$ fue un método efectivo para la inactivación de coliformes y E. coli, siendo una alternativa para el tratamiento complementario de aguas residuales domésticas de tal manera que puedan usarse con fines agrícolas.

\section{Conclusiones}

Se encontró que la combinación de sistemas biológicos y métodos no convencionales como la fotocatálisis heterogénea con $\mathrm{TiO}_{2}$ para tratar aguas residuales domésticas resulta altamente eficiente en la eliminación de microorganismos como E. coli, en comparación con métodos químicos como hipoclorito de sodio, reduciendo así el riesgo de contaminación de plantas de lechuga (Lactuca Sativa var Batavia) con bacterias entéricas.

\section{Agradecimientos}

Los investigadores agradecen al Dr. Ciro Falcony Guajardo y a las Maestras Ana Bertha Soto Guzmán y Marcela Guerrero Cruz del Centro de Investigaciones y de Estudios Avanzados del Instituto Politécnico Nacional CINVESTAV-IPN México D.F., por su colaboración en los servicios de microscopía electrónica de barrido y difracción de rayos $\mathrm{X}$.

\section{Financiación}

Este trabajo fue financiado con recursos de la oficina de Fomento a la Investigación de la Vicerrectoría Académica de la Pontificia Universidad Javeriana. Proyecto número 3404.

\section{Conflicto de intereses}

Los autores afirman no tener conflicto de intereses.

\section{Referencias}

1. Pedrero F, Kalavrouziotis I, Alarcón JJ, Koukoulakis P, Asano T. Use of treated municipal wastewater in irrigated agriculture - Review of some practices in Spain and Greece. Agriculture Water Management 2010; 97 (9): 1233-1241.

2. Gelover LA, Gelover S, Gomez LA, Reyes K, Leal MT. A practical demonstration of water disinfection using $\mathrm{TiO}_{2}$ films and sunlight. Water Research 2006; 40 (17): 3274-3280.

3. Maynard H, Ouki S, Williams C. Tertiary lagoons: A review of removal mechanisms and performance. Water Research 1999; 33 (18): 3782-3788.

4. Lubello C, Gori R, Nicese F, y Ferrini F. Municipaltreated wastewater reuse for plant nurseries irrigation. Water Research 2004; 38, 2939-2947

5. Lydakis N, Riga D, Katsivel E, Mantzavinos D, Xekoukoulotakis N. Disinfection of spring water and secondary treated municipal wastewater by $\mathrm{TiO}_{2}$ photocatalysis. Desalination 2010; 250, 351-355.

6. Rincon A, Pulgarin C. Photocatalytical inactivation of E. coli: effect of (continuous-intermittent) light intensity and of (suspended-fixed) $\mathrm{TiO}_{2}$ concentration. Applied Catalysis B: Environmental 2003; 44, 263-284.

7. Alcaldía Municipal de Subachoque (2008:Subachoque, Cundinamarca). Sistema Integral de información municipio de Subachoque [en linea]: Noviembre, 2008.Subachoque,Cundinamarca:<http:/ /www.subachoque.gov.co/index.php?sec=2\&sub=1> [Consultada: 16 ago. 2009].

8. American Public Health Association (APHA). Standard methods for examination of water and wastewater. 21th edition. American Public Health Association/American Water Works Association/Water Environment Federation. Washington, D.C., United States. 2005, 1500 p. 
9. Arango A, Rivera D, Martínez MM, Carrascal AK, Pedroza AM. Elaboración de películas de $\mathrm{TiO}_{2}$ por sedimentación para el pos-tratamiento de un efluente anaeróbico generado en un relleno sanitario. Superficie y vacio 2009; 22 (1): 10-16.

10. Rizzo L. Inactivation and injury of total coliform bacteria after primary disinfection of drinking water by $\mathrm{TiO}_{2}$ photocatalysis. Journal of Hazardous Materials 2009; 165, 48-51.

11. Palese AM, Pasquale V, Celano G. Figliuolo G, Masi S, Xiloyannis C. Irrigation of olive groves in Southern Italy with treated municipal wastewater: Effects on microbiological quality of soil and fruits. Agriculture, Ecosystems and Environment 2009; 129, 43-45.

12. Dheaya MA, Alrousan P, Dunlop SM, McMurray T, Byrne, J. Photocatalytic inactivation of E. coli in surface water using immobilized nanoparticle $\mathrm{TiO} 2$ films. Water Research 2009; 43, 47-54.

13. Rose JB, Dickson LJ, Farrah SR, Carnahan RP. Removal of pathogenic and indicator microorganisms by full-scale water reclamation facility. Water Research 1996; 30 (11): 2785-97.

14. Kayser R, Boll R, Muller HE. Quantitative determinations Finnish standard for total coliforms with of the elimination of Salmonellae by biological treatment of wastewater. Zentralblatt Bakteriologie Mikrobiologie Hygiene 1987; 184,195-205.

15. Koivunen J, Siitonen A, Heinonen-Tanski H. Elimination of enteric bacteria in biological-chemical waste- water treatment and tertiary filtration units. Water Research 2003; 37, 690-698

16. Xie YF. Disinfection Byproducts in Drinking Water: Formation, Analysis, and Control. CRC Press. Florida, United States. 2004, 161 p.

17. Takashi S, Masahito T. Enhancement of phage inactivation using photocatalytic titanium dioxide particles with different crystalline structures. Biochemical Engineering Journal 2006; 28, 303-308.

18. Baram N, Starosvetsky D, Starosvetsky J, Epshtein M, Armon R, Ein-Eli J. Enhanced inactivation of $E$. coli bacteria using immobilized porous $\mathrm{TiO}_{2}$ photoelectrocatalysis. Electrochemical Acta 2009; 54, 3381-3386

19. Nadtochenkoa VA, Nadtochenkoa AG, Rincon B, Stancaa SE, Kiwi J. Dynamics of E. coli membrane cell peroxidation during $\mathrm{TiO}_{2}$ photocatalysis studied by ATR-FTIR spectroscopy and AFM microscopy. Journal of Photochemistry and Photobiology A: Chemistry 2005; 169,131-137.

20. Christensen PA, Dilks A, Egerton TA, Temperley J. Infrared spectroscopic evaluation of the photodegradation of paint Part II: The effect of UV intensity \& wavelength on the degradation of acrylic films pigmented with titanium dioxide. Journal of Materials Science 2000; 35, 5353-5358.

21. Caballero KA, Whitehead NS, Allen J, Verran I. Inactivation of Escherichia coli on immobilized $\mathrm{TiO}_{2}$ using fluorescent Light. Journal of Photochemistry and Photobiology A: Chemistry 2009; 202, 92-98. 